KCL-MTH-99-50

December 1999

\title{
Canonical Quantization and Topological Theories
}

\author{
Talk given at QG99, Third Meeting on \\ Constrained Dynamics and Quantum Gravity, \\ Villasimius (Sardinia, Italy) September 13-17, 1999
}

\author{
Alice Rogers \\ Department of Mathematics \\ King's College \\ Strand, London WC2R 2LS, Great Britain
}

\begin{abstract}
The canonical quantization of the topological particle is described; it is shown that BRST quantization of the model gives the supersymmetric quantum mechanical model considered by Witten when investigating Morse theory, and the rigorous path integral method appropriate for this model is discussed. Possibilities for the extension of this work to two dimensional models are briefly considered.
\end{abstract}

\section{Introduction}

In this talk the first steps in a rigorous study of topological quantum theories using canonical methods is described. A topological theory is characterised by a high degree of symmetry, with the number of symmetries equal to the number of fields of the theory. As a result the system has no dynamical degrees of freedom, and the space of fields modulo symmetries, which is trivial at the linear level, is typically a finite-dimensional moduli space which encodes 'topological' information. 
As with quantum theories in general, a powerful tool for studying a topological theory is functional integration. The generic expression for the vacuum generating functional takes the form

$$
\int_{\text {fields/symmetries }} \mathcal{D} \phi \exp (i S[\phi])
$$

where $S(\phi)$ is the action of the field $\phi$. Using standard (non-rigorous) methods of quantum field theory a number of new and unexpected mathematical results have been been derived from topological models, results which in many cases have then been fully proved by more standard mathematical methods, but which would probably not have been discovered without the insights gained from the quantum field theory. (An early appearance of topological invariants in the quantum field theoretic situation is due to Belavin, Polyakov, Schwarz and Tyupin [1]. A more recent example of the powerful application of topological quantum field theory in mathematics may be found in [2], while fuller accounts of earlier work in this field may be found in the books of Nash [3] and Schwarz [4].) Most functional integrals such as (11), and related expressions with operator insertions, have not at present been properly defined. However, since these integrals have such astonishing mathematical power, it seems that an attempt to define these objects rigorously should be more than worth while. In this talk we show how this may be done for the simplest topological model, the topological particle, and describe briefly some recent work by Hrabak [5] which might lead to progress in the canonical quantization of topological field theories.

Some rigorous results on path integrals (that is, functional integrals in quantum mechanics) are known. The basic classical result (which is described by Simon in [6]) for a particle of unit mass moving in one dimension with Hamiltonian

$$
H=\frac{1}{2} p^{2}+V(x)
$$

gives the action of the imaginary time evolution operator $\exp (-H t)$ on a wave function $\psi(x)$ by the formula

$$
\exp (-H t) \psi(x)=\int d \mu \exp \left(-\int_{0}^{t} V((x(s)) d s) \psi(x(t))\right.
$$

where $d \mu$ denotes Wiener measure starting from $x$, and $x(t)$ are corresponding Brownian paths; the potential $V$ must satisfy certain analytic conditions. 
The curved space analogue of this result for a Riemannian manifold has been developed by Elworthy [7] and by Ikeda and Watanabe [8]. The expression for evolution according to the Hamiltonian $H=L+V(x)$ where $L$ is the scalar Laplacian looks identical to (3), but with $x(t)$ a process depending on metric and connection rather than simply flat space Brownian motion. Tangent space geometry plays an essential part in the theory. The present author has further extended these methods by developing a flat space theory of fermionic path integrals 99 and marrying it with Brownian motion on manifolds to give Brownian motion on supermanifolds in a suitable form for handling the Hodge-de Rham operator and the Dirac operator on manifolds 10, 11.

\section{The Topological particle}

Following Beaulieu and Singer [12] we consider the quantum-mechanical model with fields which are maps $x: I \rightarrow M$, where $I$ is the interval $[0, t]$ and $M$ is an $n$-dimensional Riemannian manifold with metric $g$. The action of the theory is

$$
S[x(.)]=\int_{0}^{t} i \omega_{\mu}\left(x\left(t^{\prime}\right)\right) \dot{x}^{\mu}\left(t^{\prime}\right) d t^{\prime}
$$

$\omega=d h$ is an exact one form on $M$ with local co-ordinate expression $\omega=$

$\omega_{\mu}(x) d x^{\mu}$ and $\dot{x}^{\mu}\left(t^{\prime}\right)=\frac{d x^{\mu}}{d t^{\prime}}$. The action can be expressed in the simpler form $S[x()]=.i(h(x(t))-h(x(0)))$ which shows that the action is indeed highly symmetric, being independent of all but the endpoints of the paths $x(t)$.

While Beaulieu and Singer consider the case where $\omega=0$, we consider the case where $h$ is a Morse function on $M$ (so that $\omega=d h$ is only zero at isolated points). To carry out the canonical quantization we first evaluate the momentum $p_{\mu}$ conjugate to $x^{\mu}$, obtaining

$$
p_{\mu}=\frac{\delta \mathcal{L}}{\delta \dot{x}^{\mu}}=i \omega_{\mu}
$$

which shows that the theory has $n$ constraints

$$
T_{\mu} \equiv p_{\mu}-i \omega_{\mu} .
$$

The Hamiltonian of the theory is as usual defined to be $H(p, x)=p_{\mu} x^{\mu}-$ $\mathcal{L}(x, \dot{x})$, so that, as is generally the case for a topological theory, the Hamiltonian of the theory (prior to gauge-fixing) is zero. Since $\omega$ is closed these constraints are first class and abelian, that is $\left\{T_{\mu}, T_{\nu}\right\}=0$ and $\left\{T_{\mu}, H_{c}\right\}=0$. 
The infinitesimal gauge transformations generated by $T_{\mu}$ are

$$
\delta_{\epsilon} \psi(x)=-i \epsilon\left(\partial_{\mu} \psi(x)+\omega_{\mu}(x) \psi(x)\right)
$$

where $\psi(x)$ is a wave function and quantization is in the Schrödinger picture with $p_{\mu}=-i \partial_{\mu}$. (Below, when ghosts are introduced, we will find that we require $p_{\mu}$ to be represented as a covariant derivative $-i \nabla_{\mu}$.) The explicit form of the gauge transformations suggests that representative of gauge equivalence classes may be obtained from the condition $X^{\mu} \psi=0$ where $X^{\mu}=g^{\mu \nu}\left(p_{\nu}+i \omega_{\nu}\right)$. The validity of these gauge-fixing conditions will become clear below.

The BRST quantization scheme will now be applied to this system; to do this anticommuting ghosts $\eta^{\mu}$ together with their conjugate momenta $\pi_{\mu}$ are introduced. (The phase space is now a $(2 n, 2 n)$-dimensional supermanifold, with odd coordinates $\eta^{\mu}, \pi_{\mu}$ transforming as indices suggest.) Poisson brackets on this extended phase space are defined by the symplectic form

$$
d p_{\mu} \wedge d x^{\mu}+\nabla \pi_{\mu} \wedge \nabla \eta^{\mu}+\frac{1}{2} d x^{\mu} \wedge d x^{\nu} R_{\mu \nu \kappa}{ }^{\lambda} \eta^{\kappa} \pi_{\lambda},
$$

(where $\nabla$ denotes covariant differentiation using the Levi-Civita connection); this is a special case of the symplectic form introduced by Rothstein [13]. Quantization is carried out by introducing states represented by wave functions $\psi(x, \eta)$ and momenta acting as $p_{\mu}=-i \nabla_{\mu}, \pi_{\mu}=-i \frac{\partial}{\partial \eta^{\mu}}$. The wave functions $\psi(x, \eta)$ are functions on the $(n, n)$-dimensional supermanifold $S M$ with local coordinates $x^{\mu}, \eta^{\mu}$, and the explicit form of the action of the covariant derivative on a wave function is given by

$$
\nabla_{\mu} \psi(x, \eta)=\partial_{\mu} \psi(x, \eta)+\Gamma_{\mu \nu}^{\lambda} \eta^{\nu} \frac{\partial}{\partial \eta^{\lambda}} \psi(x, \eta) .
$$

The BRST charge $Q$ takes the standard form $Q=\eta^{\mu} T_{\mu}=-i \eta^{\mu}\left(\partial_{\mu}+\right.$ $\omega$ ). (A covariant derivative is not required here because of the symmetry of the connection.) The gauge-fixing fermion $\chi$ also takes the standard form $\chi=\pi_{\mu} X^{\mu}=-i g^{\mu \nu} \pi_{\mu}\left(\nabla_{\nu}-\omega_{\nu}\right)$. Alternatively, if we make the natural identification of forms on $M$ with wave functions $\psi(x, \eta)$ then $Q=-i e^{-h} d e^{h}$, $\chi=e^{h} \delta e^{-h}$ where $d$ denotes exterior differentiation of forms and $\delta=* d *$ is the adjoint operator. The gauge-fixing Hamiltonian is then

$$
\begin{aligned}
H_{g} & =i(Q \chi+\chi Q) \\
& =d \delta+\delta d+g^{\mu \nu} \omega_{\mu} \omega_{\nu}-i\left(\pi_{\mu} \eta^{\nu}-\eta^{\nu} \pi_{\mu}\right) \frac{\partial^{2} h}{\partial x^{\mu} \partial x_{\nu}} .
\end{aligned}
$$


This Hamiltonian has appeared in the literature on other occasions; for instance, when $h$ is constant, it is the Hamiltonian used by Alvarez-Gaumé 114 to prove the Atiyah-Singer index theorem. (A rigorous version of this proof may be found in [10].) It is also the first supersymmetric Hamiltonian used by Witten in his study of Morse theory 15.

It is evident that the choice $\chi=\pi_{\mu} X^{\mu}$ is a good gauge-fixing condition, satisfying the essential conditions derived by the author in [16]. First, as observed by Beaulieu and Singer in the constant $h$ case, the standard theory of harmonic forms shows that the gauge condition determines a unique element of each $Q$ cohomology class. (The observation that these arguments extend to all functions $h$ is due to Witten [15.) Also, the zeros of $H_{g}$ coincide with these representatives of the cohomology classes, while the eigenvalues of $H_{g}$ tend to infinity, so that this Hamiltonian does regulate the non-physical states.

The path integral formulae for this Hamiltonian can be put in rigorous form using the methods of the author in [10, 11, and used to establish rigorous results for this model. The key idea in this approach is to use Brownian paths $x_{t}, \eta_{t}$ in the supermanifold $S M$ with local coordinates $x^{\mu}, \eta^{\mu}$. The Brownian paths are defined by the stochastic differential equations

$$
\begin{aligned}
x_{t}^{\mu} & =x^{\mu}+\int_{0}^{t} e_{a, s}^{\mu} \circ d b_{s}^{a} \\
e_{a, t}^{\mu} & =e_{a}^{\mu}+\int_{0}^{t}-e_{a, s}^{\nu} e_{b, s}^{\lambda} \Gamma_{\nu \lambda}^{\mu}\left(x_{s}\right) \circ d b_{s}^{b} \\
\eta_{t}^{\mu} & =\eta^{\mu}+\theta_{t}^{a} e_{a, t}^{\mu} \\
+ & \int_{0}^{t}\left(-\eta_{s}^{\nu} \Gamma_{\nu \lambda}^{\mu} e_{b, s}^{\lambda} \circ d b_{s}^{b}-\theta_{t}^{a} d e_{a, s}^{\mu}+\frac{1}{4} \eta_{s}^{\nu} R_{\nu \lambda \kappa}{ }^{\mu}\left(x_{s}\right) \eta_{s}^{\lambda} \rho_{s}^{a} e_{a, s}^{\kappa} d s\right),
\end{aligned}
$$

where $b_{t}$ is flat bosonic Brownian motion and $\left(\theta_{t}, \rho_{t}\right)$ is flat fermionic Brownian motion. The measure corresponding to this process incorporates as the 'kinetic term' the heat kernel of the Laplace-Beltrami operator $(d+\delta)^{2}$, so that these Brownian paths are appropriate for the analysis of the Hamiltonian (10). Further details will be found in [17], where it will be shown that Witten's approach to Morse theory [15] can be put on an entirely rigorous mathematical footing. (Some parts of Witten's analysis have been proved rigorously by Simon et al [18] and by Mathai and Wu [19]; however the explicit modeling of the manifold's cohomology via critical points and instanton calculations does not appear to have received a full mathematical treatment.) 


\section{The two-dimensional topological sigma model}

To conclude, a brief indication of some developments in a two-dimensional topological model will be described. The model, which was first proposed by Witten [20], concerns the geometry of $J$-holomorphic curves (or pseudoholomorphic maps) $u: \Sigma \rightarrow M$ from a Riemann surface $\Sigma$ into an a $2 m$-dimensional almost-Kaehler manifold $M$. The other fields of the theory are a bosonic set $H_{\mu}^{\alpha}$ and two fermionic sets $\eta^{\mu}$ and $\pi_{\mu}^{\alpha}$. (Here $\alpha=1,2$ are indices on $\Sigma$ while $\mu=1, \ldots, 2 m$ are indices on $M$.) The fields $H$ and $\pi$ satisfy constraints $P^{-} H=0, P^{-} \pi=0$ where $P_{\beta \nu}^{-\alpha \mu}=\delta_{\beta}^{\alpha} \delta_{\nu}^{\mu}-\epsilon_{\beta}^{\alpha} J_{\nu}^{\mu}$ is a projection operator with $\epsilon$ the complex structure on $\Sigma$ and $J$ the almost complex structures on $M$. Witten constructs by hand a set of supersymmetry transformations (beginning with $\delta u^{\mu}=i \epsilon \eta^{\mu}$ ) on these fields, and then an invariant action. The model is used to derive deep geometric insights into the moduli space of $J$-holomorphic curves on $M$. Recent work of Hrabak [5] shows that the rather complicated and seemingly ad hoc supersymmetry transformations of the model can be derived in the canonical setting as BRST transformations; the novel feature of Hrabak's work is that the formalism used is not the standard canonical formalism (in which time plays a special rôle) but the multisymplectic formalism which is manifestly covariant; corresponding to the fields $u: \Sigma \rightarrow M$ there are multimomenta $p_{\mu}^{\alpha}$ (which after projection relate to Witten's $H_{\mu}^{\alpha}$ ), while in the ghost sector the ghosts have momenta $\mathcal{P}_{\mu}^{\alpha}$ which relate to Witten's $\pi_{\mu}^{\alpha}$. The BRST symmetry obtained by Hrabak corresponds directly to the $J$-holomorphicity of the embeddings. Recent work by Kanatchikov [21] on quantization in the multisymplectic framework suggests that it may be possible to use Hrabak's approach to carry out a full canonical quantization of Witten's interesting two-dimensional topological model.

\section{References}

[1] A.A. Belavin, A.M. Polyakov, A.S. Schwarz, and Yu S. Tyupin. Physics Letters, 59B:85, 1975.

[2] E. Witten. Monopoles and four manifolds. Math. Res. Lett., 1, 1994.

[3] C. Nash. Differential topology and quantum field theory. Academic Press, 1991. 
[4] A. Schwarz. Quantum Field Theory and Topology. Springer, 1993.

[5] S.P. Hrabak. On the multisymplectic origin of the nonabelian deformation algebra of pseudoholomorphic embeddings into strictly almost kahler ambient manifolds, and the corresponding BRST algebra. Preprint mathph/9904026. 1999.

[6] B. Simon. Functional Integration and Quantum mechanics. Academic Press, 1979.

[7] K.D. Elworthy. Stochastic Differential Equations on Manifolds. London Mathematical Society Lecture Notes in Mathematics. Cambridge University Press, 1982.

[8] N. Ikeda and S. Watanabe. Stochastic differential equations and diffusion processes. North-Holland, 1981.

[9] A. Rogers. Fermionic path integration and Grassmann Brownian motion. Communications in Mathematical Physics, 113:353-368, 1987.

[10] A. Rogers. Stochastic calculus in superspace II: differential forms, supermanifolds and the Atiyah-Singer index theorem. Journal of Physics A, 25:6043-6062, 1992.

[11] Alice Rogers. Path integration, anticommuting variables and supersymmetry. Journal of Mathematical Physics, 36:2531-2545, 1995.

[12] Beaulieu and I. Singer. The topological sigma model. Commun. Math. Phys., 125:227-237, 1989.

[13] M. Rothstein. The structure of supersymplectic supermanifolds. In C. Bartocci, U. Bruzzo, and R. Cianci, editors, Differential geometric methods in theoretical physics, proceedings, Rapallo 1990, volume 375 of Lecture Notes in Physics, pages 331-343. Springer, 1991.

[14] L. Alvarez-Gaumé. Supersymmetry and the Atiyah-Singer index theorem. Comm. Math. Phys., 90:161-173, 1983.

[15] E. Witten. Supersymmetry and Morse theory. Journal of Differential Geometry, 17:661-692, 1982. 
[16] A. Rogers. Gauge fixing and BFV quantization. Classical and Quantum Gravity, to appear.

[17] A. Rogers. The topological particle. (In preparation.)

[18] H.L. Cycon, R.G. Froese, W. Kirsch, and B. Simon. Schrödinger operators. Springer, 1987.

[19] S. Wu and V. Mathai. Equivariant holomorphic morse inequalities. 1: A heat kernel proof. ICTP preprint IC/96/29, 96.

[20] E. Witten. Topological sigma models. Commun.Math.Phys., 118:411, 1988.

[21] I. V. Kanatchikov. On quantization of field theories in polymomentum variables. To be published in the proceedings of International Conference on Particles, Fields and Gravitation (Devoted to the Memory of Professor Ryszard Raczka), Lodz, Poland, 15-18 Apr 1998., AIP Proceedings 1998 hep-th/9811016. 